\title{
Mathematical Model of Enterprise with Revolving Funds Deficit: Analysis of Demand Shocks 2020
}

\author{
D. A. Alimov ${ }^{1,2 *}$, N. K. Obrosova ${ }^{1,2,3^{* * *}}$, and A. A. Shananin ${ }^{1,2,3,4^{* * * *}}$ \\ (Submitted by A. V. Lapin) \\ ${ }^{1}$ Lomonosov Moscow State University, Moscow, 119991 Russia \\ ${ }^{2}$ Moscow Institute of Physics and Technology (State University), \\ Dolgoprudny, Moscow oblast, 141701 Russia \\ ${ }^{3}$ Dorodnicyn Computing Centre of Federal Research Center "Computer Science and Control," \\ Russian Academy of Sciences, Moscow, 119333 Russia \\ ${ }^{4}$ Peoples Friendship University of Russia, Moscow, 117198 Russia \\ Received May 22, 2020; revised May 31, 2020; accepted June 10, 2020
}

\begin{abstract}
Russian enterprises of manufacturing complex are facing financial crisis because of the dramatic drop in demand for products due to the pandemic. Since a significant part of the population is employed in this sector, measures to support production in manufacturing industries are discussed at various levels. Based on the mathematical model of production, taking into account unstable demand, we propose a methodology for analyzing the effectiveness of support measures. Financial performance of the enterprise are calculated using an analytical solution of the Bellman equation that determines the valuation of the company, as well as the ergodicity property of the random process of product sales. The application of the methodology is illustrated by the example of KAMAZ Group.
\end{abstract}

DOI: $10.1134 / \mathrm{S} 1995080220120045$

Keywords and phrases: Bellman equation, Production model, stochastic Poisson flow, identification, company's Value, Houthakker-Johansen model, unstable demand, current assets deficit.

\section{INTRODUCTION}

Currently the public attention is focused on COVID-19 pandemic and its economic impact. For the moment the long-term consequences and structural changes caused by risk globalization that the world economy is facing now can be analyzed only in most general terms. But we also need to react to shortterm economic effects caused by the pandemic and their impact on mid-term possibilities. Particularly, Russian experts highlight the following short-term economic risks:

- decrease of household incomes, purchasing power and effective demand;

- instability in realization of services and manufactured goods that results in shortages of working capital;

- increase of manufacturers' credit debts, increase of production costs due to forced downtime while having to pay constant costs and increase of foreign material costs due to increase of dual currency basket rate;

\footnotetext{
${ }^{*}$ E-mail: alimov2007d@gmail.com

${ }^{* *}$ E-mail: nobrosova@yandex.ru

${ }^{* * *}$ E-mail: alexshan@yandex.ru
} 
- possibility of mass bankruptcy of labor-intensive industry companies and the growth of unemployment rate.

These threats should be responded to by traditional state support measures provided to manufacturing facilities:

- subsidies of interest rates on loans;

- tax deferrals and vacations to manufacturers;

- direct subsidies to manufacturers with partial transfer of property rights to the state;

- support of demand for goods and services by public orders.

The determination of the size and forms of state support measures should be based on the economic health of enterprises and industry specifics. The scale of the problem makes it difficult to use traditional methods aimed at solving forecasting problems under relatively stable economic situations. Therefore, one of the pressing challenges is to develop improved methods of analysis that allow to evaluate the the enterprises health and determine support measures for manufacturing complexes that are functioning under conditions of irregular demand, shortages of working capital and credit debt growth. Short-term and medium-term assessments of production capacities are traditionally based on mathematical models that do not take into account the instability of realization of products (see [1-3]). This is based on the assumption that a sufficient volume of the enterprise's current assets makes it possible to neutralize the impact of delays in realization of products. However, if product realization time expectation is comparable to its standard deviation, such assumption loses its point. We developed a model [4-6] for evaluating indicators for manufacturing under conditions of irregular demand and shortage of working capital. In the paper [7] we studied how credit debts and bankruptcy proceedings impact these indicators. We showed in [8] on the example of automotive industry enterprises that the developed mathematical model can be used to analyze indicators for manufacturing under conditions of shortage of working capital. In this paper we apply our method to analyze the situation and the efficiency of manufacturing support measures under conditions of the economic crisis caused by the COVID-19 pandemic.

\section{MODEL, BASIC ASSUMPTIONS AND METHOD DESCRIPTION}

The main consequence of the pandemic for manufacturing enterprises is an increase in instability and a drop in demand for manufactured products. It leads to the deficit of working capital increasing. We apply the model to analyze the functioning of companies in the current economic environment, taking into account the volatility of demand, the influence of the trade infrastructure and debt load. The model allows analysing the influence of the parameters of the economic conjuncture on the assessment of the company's value and can be used to assess the creditworthiness of companies, operating under conditions of current assets deficit and unstable demand in a quasi-stable economic situation. Here we discuss the basic elements of our model. We use the following basic assumptions

- we consider a production of a uniform product with unit cost $y$ and product price $p$; the full enterprise capacity is $\eta$;

- the moments of realization of products form a Poisson flow with the parameter $\lambda(1 /$ year) cause its mathematical expectation compares to the standard deviation (the instability of product sales);

- there exists the upper bound $Y^{*}$ on the maximum volume of a single sale of goods (restriction of trade infrastructure);

- company's value $V\left(Y_{0}, L_{0}\right)$ is a function on current inventories in stock $Y_{0}$ and a current company's long-term debt $L_{0}$;

- the replenishment of current assets is possible by a short-term credit line $K(t)$ at interest rate $r$ in a competitive market only; 
- production runs on a full capacity $\eta$ by a credit line $K(t)$ during the time period $\tau$, that chooses the owner of the enterprise; if there is no sale to the moment $\tau$ the production stops until the moment of the sale; so the average capacity utilization can be less than 1.

- at the time of the product sale the enterprise's owner receives revenue, pays for loans and applies for the short-term credit line $K(t)$ again;

- if the income of one sale is not enough to pay for loans, then the short-term debt is restructured to a long-term one with the interest rate $\Delta$;

- the sale profit after pay for loans is withdrown from current assets by the owner;

- the short-time credit is used to the current assets replenishment only.

We assume that the activities of the manufacturer are described by processes occurring at different temporal scales. Changes in production capacity $\eta$ and trade infrastructure, characterized by the value of $Y^{*}$, occur in slow time. A change in market conditions, which determines the price $p$, cost $y$, interest rate $r$ and frequency of sales of products $\lambda$, occurs at medium temporal scale. On a fast temporal scale operational decisions are made about the current production volumes that are determined by the period of use of the loan. Consider the functioning of production in a fast time between two consecutive moments of sales. Under our assumptions

$$
K(t)=y \eta \theta(\tau-t), \quad \text { where } \theta(x)=\left\{\begin{array}{l}
1, x>0 \\
0, x \leqslant 0
\end{array}\right.
$$

The output value at the time $t$ is

$$
\frac{d Y(t)}{d t}=\frac{1}{y} K(t), \quad Y(0)=Y_{0},
$$

that is

$$
Y(t)=Y_{0}+\eta \min \{t, \tau\}
$$

We assume that the total debt of the company $L(t)$ is divided into two parts $L_{1}, L_{0}$, with interest rates $r$ and $\Delta$ respectively. The first part $L_{1}(t)$ corresponds to short-term debt, that is paid immediately after the sale of products by the company. The change of the second part $L_{0}$ occurs from those part of short-time debt that was not paid at the moment of product sale. I.e. the not paid short-term debt is restructured to the part of long-term loan at the interest rate $\Delta$. Consequently

$$
\left\{\begin{array}{l}
\frac{d L_{1}(t)}{d t}=K(t)+r L_{1}(t), \\
L_{1}(0)=0
\end{array}\right.
$$

and total debt is

$$
L(t)=L_{1}(t)+L_{0} e^{\Delta t}=\frac{y \eta}{r}\left(e^{r t}-e^{r(t-\tau)_{+}}\right)+L_{0} e^{\Delta t} .
$$

The goal of the owner of production is to choose the time period $\tau$, during which production costs are advanced by the credit line, in order to maximize the mathematical expectation of the sales income $V\left(Y_{0}, L_{0}\right)$, discounted with the coefficient $\Delta>0$ :

$$
\begin{aligned}
& V\left(Y_{0}, L_{0}\right)=\sup _{\tau \geqslant 0} \int_{0}^{+\infty} \lambda e^{-(\lambda+\Delta) t}\left[\left(p \min \left(Y(t), Y^{*}\right)-L(t)\right)_{+}\right. \\
& \left.\quad+V\left(\left(Y(t)-Y^{*}\right)_{+},\left(L(t)-p \min \left(Y(t), Y^{*}\right)\right)_{+}\right)\right] d t .
\end{aligned}
$$

The solution of Bellman equation ( 3$) V\left(Y_{0}, L_{0}\right)$ characterizes the estimation of the value of enterprise together with the unrealized inventories of products $Y_{0}$ and accumulated debts $L_{0}$ depending on 
production indicators of company's activities and parameters of economic environment (interest rate, price, cost, etc).

Theorem 1. Let $W\left(Y_{0}\right)$ be a solution of the following Bellman equation

$$
\begin{aligned}
& W\left(Y_{0}\right)=\sup _{\tau \geqslant 0} \int_{0}^{+\infty} \lambda e^{-(\lambda+\Delta) t}\left[p \min \left(Y_{0}+\eta \min (t, \tau), Y^{*}\right)\right. \\
& \left.-\frac{y \eta}{r}\left(e^{r t}-e^{r(t-\tau)_{+}}\right)+W\left(\left(Y_{0}+\eta \min (t, \tau)-Y^{*}\right)_{+}\right)\right] d t .
\end{aligned}
$$

Then

$$
V\left(Y_{0}, L_{0}\right)=W\left(Y_{0}\right)-L_{0}
$$

is a solution of Bellman equation (3).

Proof. We substitute the function (5) into the right part of the equation (3):

$$
\begin{gathered}
\sup _{\tau \geqslant 0} \int_{0}^{+\infty} \lambda e^{-(\lambda+\Delta) t}\left[p \min \left(Y_{0}+\eta \min (t, \tau), Y^{*}\right)\right. \\
\left.-\frac{y \eta}{r}\left(e^{r t}-e^{r(t-\tau)_{+}}\right)+W\left(\left(Y_{0}+\eta \min (t, \tau)-Y^{*}\right)_{+}\right)-L_{0} e^{\Delta t}\right] d t \\
=\sup _{\tau \geqslant 0} \int_{0}^{+\infty} \lambda e^{-(\lambda+\Delta) t}\left[p \min \left(Y_{0}+\eta \min (t, \tau), Y^{*}\right)\right. \\
\left.-\frac{y \eta}{r}\left(e^{r t}-e^{r(t-\tau)_{+}}\right)+W\left(\left(Y_{0}+\eta \min (t, \tau)-Y^{*}\right)_{+}\right)\right] d t-L_{0}=W\left(Y_{0}\right)-L_{0}
\end{gathered}
$$

since $W\left(Y_{0}\right)$ is the solution of the equation (4). Consequently, the statement of the theorem is obtained.

We give a brief overview of the results of the Bellman equation (4) analysis. In [5] we prove that if the conditions of profitability are fulfilled $\lambda+\Delta>r, Y^{*}>0, p>y \frac{\lambda+\Delta}{\lambda+\Delta-r}$ equation (4) has a unique solution in the class of continuous, non-negative, nondecreasing, concave functions bounded together with its derivative on the half-open interval $[0,+\infty)$. We find the explicit form of the solution of equation (4). The optimal behavior of the manufacturer corresponding to the solution is described as follows: in the absence of product realization, production runs at full capacity at the expense of a loan, until the inventories amount $\left(\zeta_{0}+1\right) Y^{*}$ is reached, and then stops until the moment of sale. The solution of the Bellman equation allows one to determine (see [5]):

- characteristic of maximum inventories $\zeta_{0}$ as a function of model parameters,

- the ratio of the value of the company to current assets $\frac{\lambda\left(W\left(Y_{0}\right)-L_{0}\right)}{y \eta}$ as a function of model parameters, $Y_{0}, L_{0}$ and the variable $\zeta_{0}$.

The official reporting of companies contains the average indicators of their activity. Therefore, the solution of the identification problem requires the calculation of the average company performance indicators in terms of the model. To interpret model indicators in terms of official statistics, which provides time-averaged data, we propose a technique for calculating production performance in the model-averaging over the final distribution of the probabilities of the inventories dynamics. Based on ergodicity of the stochastic process of inventories dynamics, explicit expressions are obtained for the average capacity utilization $u$, the average inventories $Q$ and the average short-term debt of the company $\hat{L_{1}}$ (see $\left.[6,8]\right)$. The average short-term debt $\hat{L_{1}}$ can be interpreted as working capital of the company. The change in the company's working capital due to the assumption of restructuring of short-term debts into long-term ones equals the change in long-term debts. The results of solving the Bellman equation and analysing the random process of inventories dynamics make it possible to write out a system of model equations (three algebraic equations) whose exogenous (input) and endogenous variables are interpretable in terms of official statistics. The base point of the model in the selected year is defined by the following endogenous (evaluated by the system of the model) variables: 
- $\lambda$-characteristic of market conditions (parameter of the Poisson process),

- $\zeta_{0}$-characteristic of maximum volume of inventories,

- $\frac{\lambda Y^{*}}{\eta}$-characteristic of production potentialities taking into account the restriction of trade infrastructure.

A set of exogenous parameters of the system of the model equations that is observed by official statistics:

- $r$-short-term loans interest rate,

- $\Delta$-long-term loans interest rate,

- $R$-parameter of profitability of production that is defined by the formula

$$
R=\frac{p-y \frac{\lambda+\Delta}{\lambda+\Delta-r}}{p},
$$

- $u$-average capacity utilization rate,

- $\frac{y Q}{y u \eta}$-inventory turnover.

The system of equations for the range $0<\zeta_{0} \leq 1$ is the following

$$
\begin{gathered}
\left(\frac{1}{R}-\frac{\lambda+\Delta}{\lambda} \frac{1-R}{R}\right)\left[\frac{\lambda}{\lambda+\Delta}\left(1-e^{-\frac{\lambda+\Delta}{\lambda} \zeta_{0} \frac{\lambda Y^{*}}{\eta}}\right)+e^{-\frac{\lambda+\Delta}{\lambda} \zeta_{0} \frac{\lambda Y^{*}}{\eta}}\left(e^{\frac{\lambda+\Delta}{\lambda} \frac{\lambda Y^{*}}{\eta}}-\zeta_{0} \frac{\lambda Y^{*}}{\eta}\right)\right]=1, \\
u=1-\frac{e^{-\frac{\lambda Y^{*}\left(\zeta_{0}+1\right)}{\eta}}}{1-\frac{\lambda Y^{*}}{\eta} \zeta_{0} e^{-\frac{\lambda Y^{*}}{\eta}}}, \\
\lambda=\frac{y u \eta}{y Q}\left(2-\frac{1-\left(\lambda Y^{*} \zeta_{0} e^{\frac{\lambda Y^{*}}{\eta}}\right) / \eta-e^{-\frac{\lambda Y^{*}}{\eta}}}{1-\left(\lambda Y^{*} \zeta_{0} e^{-\frac{\lambda Y^{*}}{\eta}}\right) / \eta-e^{-\frac{\lambda Y^{*}}{\eta}\left(\zeta_{0}+1\right)}}\right)=\lambda\left(\zeta_{0}, \frac{\lambda Y^{*}}{\eta}\right) .
\end{gathered}
$$

We substitute (9) in (7) and obtain a system of two equations (7) and (8) with variables $\zeta_{0}, \frac{\lambda Y^{*}}{\eta}$. Solving this system, from (9) we find $\lambda=\lambda\left(\zeta_{0}, \frac{\lambda Y^{*}}{\eta}\right)$. Thus, from the equations system (7)-(9) we find the base point of the model $\zeta_{0}, \frac{\lambda Y^{*}}{\eta}, \lambda$. Further, we can calculate the ratio $\frac{\lambda W\left(Y_{0}\right)}{y \eta}$ that characterises the ratio of the company's valuation to the current assets. It is shown in [5] that in the case $0<Y_{0}<Y^{*}$ :

$$
\begin{gathered}
\frac{\lambda W\left(Y_{0}\right)}{y \eta}=\frac{p}{y} \frac{\lambda}{\lambda+\Delta} \frac{\lambda Y_{0}}{\eta}-\left(\frac{\lambda}{\lambda+\Delta}\right)^{2}\left[\frac{p}{y}-\frac{\lambda+\Delta}{\lambda+\Delta-r}\right]\left[e^{(\lambda+\Delta) \frac{Y_{0}}{\eta}}-1\right] \\
+\frac{\lambda}{\lambda+\Delta} \frac{\lambda W(0)}{y \eta}\left[1+\frac{\Delta}{\lambda} e^{(\lambda+\Delta) \frac{Y_{0}}{\eta}}\right]
\end{gathered}
$$

where

$$
\frac{\lambda W(0)}{y \eta}=\frac{\lambda}{\Delta}\left[\frac{\lambda}{\lambda+\Delta} \frac{p}{y}-\frac{\lambda}{\lambda+\Delta-r}-\left(\frac{\lambda}{\lambda+\Delta} \frac{p}{y}-\frac{\lambda+\Delta}{\lambda+\Delta-r}\right) \times e^{-\frac{\lambda+\Delta}{\lambda} \zeta_{0} \frac{\lambda Y^{*}}{\eta}}\right]
$$

This indicator reflects the investment attractiveness of the company in the medium term and allows one to analyse its market position. In [8] we calculate a closed-form expression for the average short-term 
loans of the company. In the case $0 \leqslant \zeta_{0}<1$ the average value of short-term loans satisfies the following expression

$$
\begin{gathered}
\frac{\lambda \hat{L_{1}}\left(r, \zeta_{0}\right)}{y u \eta}=\frac{\lambda}{\lambda-r} \frac{1-u}{u}\left[e^{\frac{\lambda Y^{*}}{\eta}\left(\zeta_{0}+1\right)}+e^{-\frac{\lambda Y^{*}}{\eta}}\left(\frac{1}{2}\left(\frac{\lambda Y^{*}}{\eta} \zeta_{0}\right)^{2}+\frac{\lambda Y^{*}}{\eta} \zeta_{0}\right)-\frac{\lambda Y^{*}}{\eta} \zeta_{0} e^{\frac{\lambda Y^{*}}{\eta} \zeta_{0}}\right. \\
\left.-\frac{\lambda Y^{*}}{\eta}\left(\zeta_{0}+1\right)-1\right] .
\end{gathered}
$$

In [9] we propose a technique of identifying of exogenous parameters of the developed model based on the analysis of statistical reporting of companies. We can construct various scenarios of predictive evaluations in terms of the model by methods of comparative statics. To do this we can fix one part of parameters at the base point and analize the dependence of enterprise's indicators from the other part of parameters, that change in connection to chosen scenario. For example if we know the expert assessment of changes in demand for the company's products $\lambda$, then we can calculate the capacity utilization $u$ as an output parameter. The capacity utilization is an important indicator, as it affects not only the economic performance of the company, but also the solvency of the population. With reduced capacity utilization, there is usually a reduction in staff time and payroll.

\section{SCENARIO ANALYSIS OF GOVERNMENT FINANCIAL SUPPORT FOR AUTOMOTIVE INDUSTRY DURING CORONAVIRUS CRISIS IN 2020 BY THE EXAMPLE OF KAMAZ GROUP}

The developed production model allows to estimate the economic efficiency of government support measures to the manufacturing sector and to analyse probable short- and medium-term impacts of demand shocks and debt burden in the absence of so measures.

The methodology of predictive calculations using the developed model is based on the approach of comparative statics. We illustrate the application of this approach by the example of the impact evaluation of changes in demand on company products. Having calculated the base point of the model by the equation system (7)-(9) on the basis of the statistical reporting data of the company and the current economic situation, we assume that in the medium-term horizon, the structural characteristic of demand $Y^{*} / \eta$ changes insignificantly. Then the change in demand corresponds to a proportional change in the frequency of product sales $\lambda$ in the model. We will also assume that the structure of selling prices and unit costs $p / y$ will remain at the basic level in the near future (alternative scenarios may take into account rising prices for resources and manufactured products). Then the model equations (6) $-(8)$ make it possible to calculate the characteristics of the company with a scenario shift of the system base point $\zeta_{0}, \frac{\lambda Y^{*}}{\eta}, \frac{\lambda W\left(Y_{0}\right)}{y \eta}(10)$ with a change in $\lambda$ and the specified values of the interest rate $r$ and the owner income discount $\Delta$. Further, from the relations (8), (10), (11), we can calculate the main indicators of production activity in conditions of changed demand.

The enterprises of the automotive industry are the typical representatives of the Russian manufacturers working in conditions of fierce competition with imports. Using the model, we carried out in [8] an analysis of the economic operating conditions (for the period 2011-2016) of KAMAZ, a large cityforming enterprise that occupies a significant share of the trucking market in Russia and whose position affects the social stability of the whole region. The KAMAZ company can count on targeted measures of state support during the crisis. Standard support measures include interest rates subsidies on loans and state orders to support demand. Such measures contributed to the stabilization of the company performance during the 2014 crisis, accompanied by an increase in interest rates on loans and a decline in effective demand in the face of a weakening ruble. A detailed analysis of the effectiveness of these measures using the developed model we presented in [8].

When carrying out calculations according to the model for KAMAZ we propose to consider the value $\frac{\lambda W(0)}{y \eta}(10)$ as an estimation of the total value of the company $\frac{\lambda\left(W\left(Y_{0}\right)-L_{0}\right)}{y \eta}$ (see the Theorem 1 . We calculate the value $\frac{\lambda W(0)}{y \eta}$ on the bas point of the model based on the official statistical reports of the company for the selected year. This approximation is valid due to two circumstances. Firstly, stocks of finished goods and products in progress of the company do not significantly affect the dynamics of the total value of the KAMAZ (Fig. 1). Secondly, the available statistical data does not allow to separate 


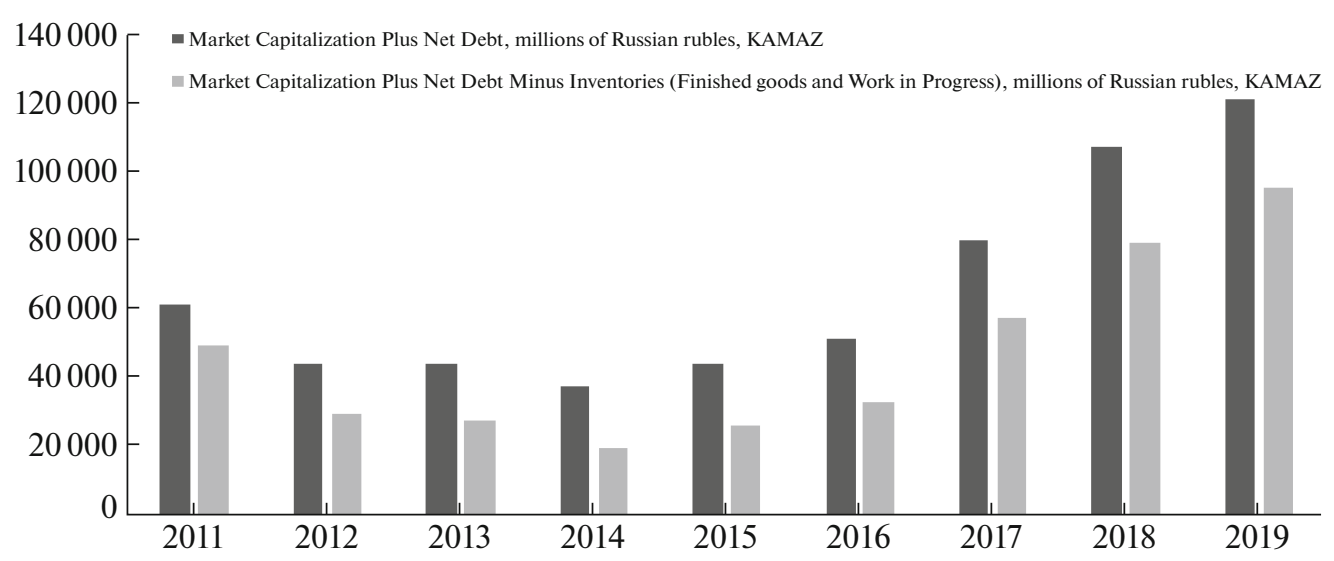

Fig. 1.
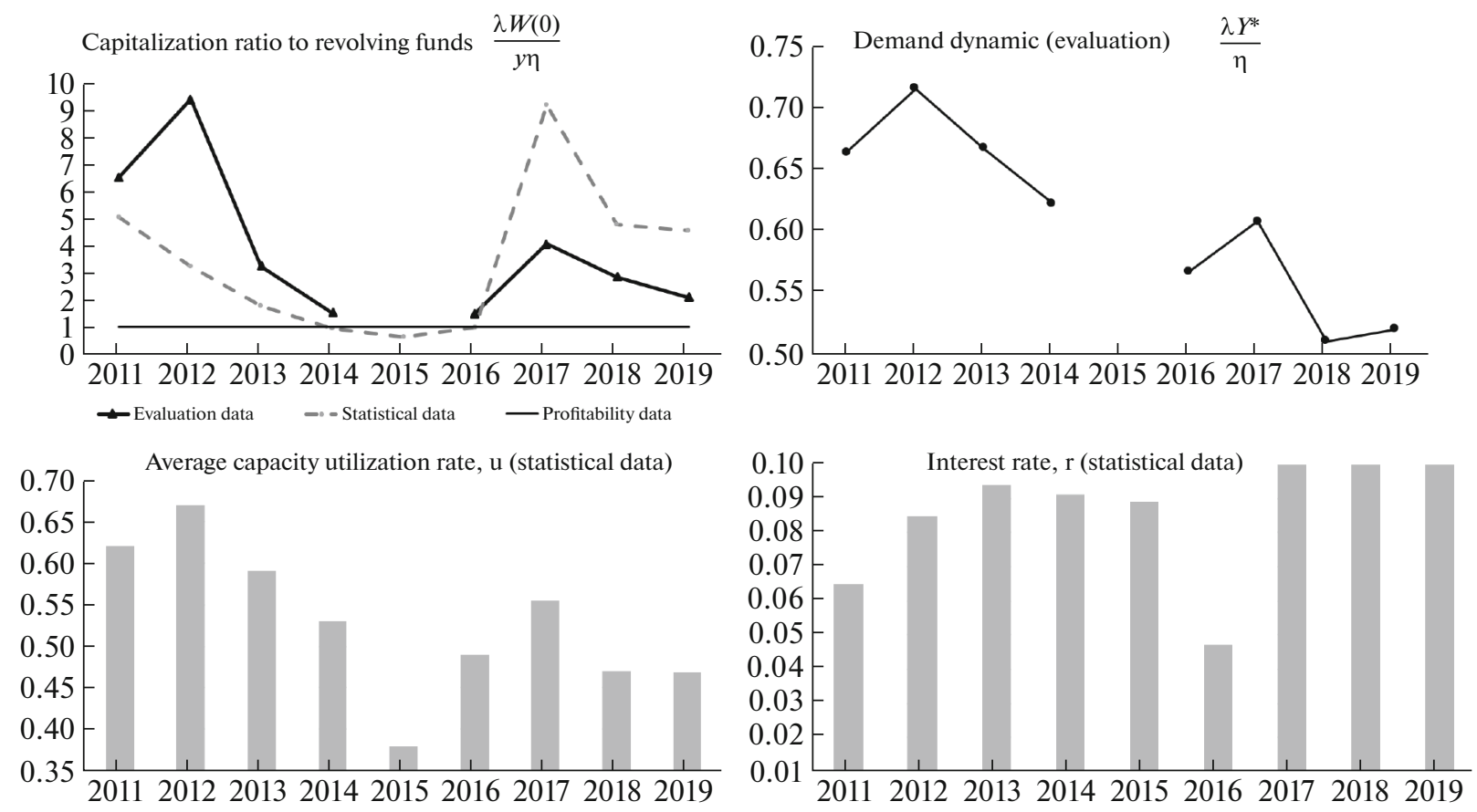

Fig. 2.

the interest payment flows on long-term and short-term loans of the company. Therefore, we take into account the corresponding total stream of interest payments when calculate the profitability ratio (6) by the model identification. Thus, the calculated value of $W(0)$ actually takes into account the debt payments of the company and corresponds to the real cash flow generated by the company after interest payments.

Figure 2 shows the updated calculation results that we obtained based on the identification of the model according to the official reporting of the company 2011-2019. The solid line in the first graph corresponds to the valuation of the company in relation to revolving funds calculated using the model and reflects the real indicators of its activity in the period under review (in 2015, the company was unprofitable and calculation by the model is not possible). The dashed line corresponds to the market value of the company (market capitalization, statistics) and includes the speculative component of value (the reasons for the discrepancy between these curves are analyzed in [8]).

Decrease in demand as a result of the crisis of 2014 led to unprofitability of the enterprise and the absence of urgent measures could lead to bankruptcy of the company (Fig. 2). Calculations show that the company successfully overcame the 2014-2015 profitability crisis and that was a result 


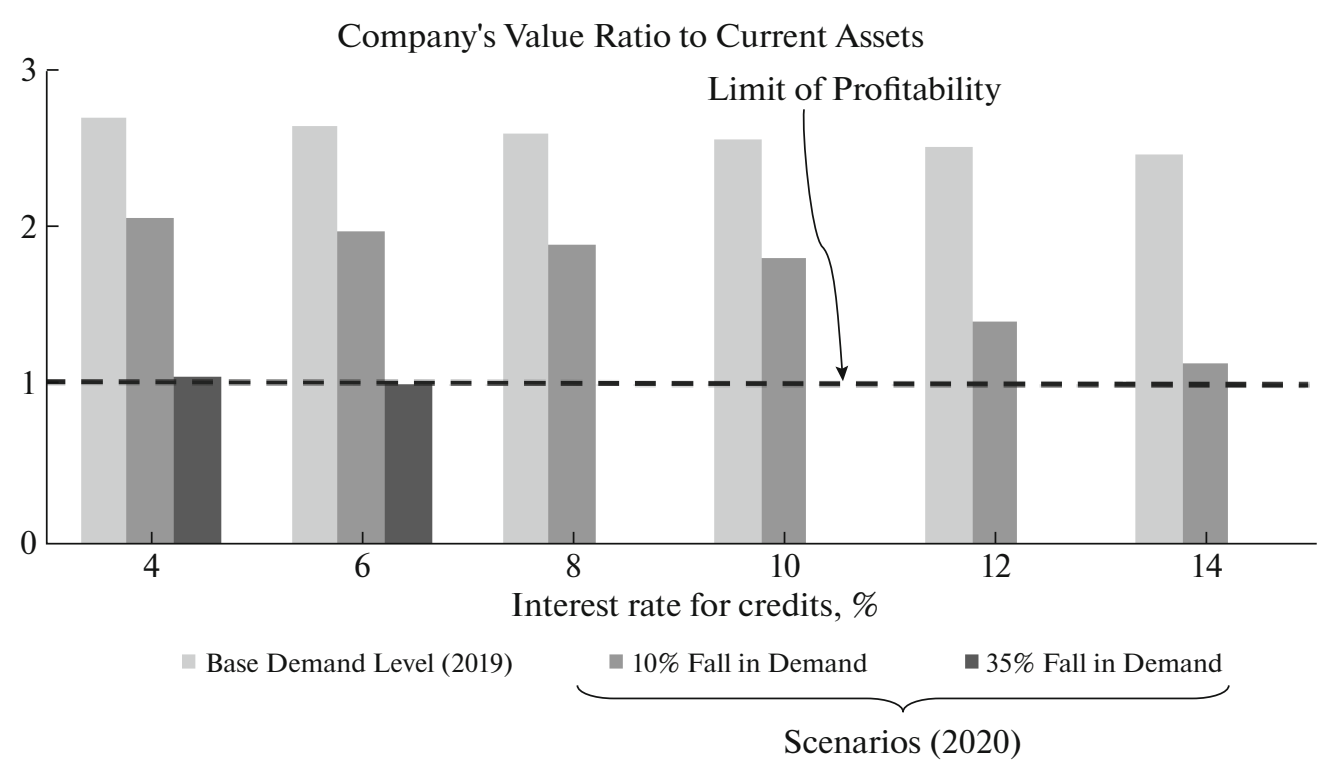

Fig. 3.

of subsidizing interest rates in 2016 and increased demand through large government orders. The measures of the government turned out to be effective, the company's position improved significantly in 2017 and stabilized in 2018-2019, despite the growth of the real interest rate to the pre-crisis level and a slight decrease in demand (Fig. 2).

The crisis of 2020 caused by COVID-19 may again exacerbate problems of the company's survival. A retrospective analysis of the KAMAZ's indicators (Fig. 2) shows that the main threats in this situation are a drop in demand and an increase in the debt burden due to an increase in the interest rate on company loans. According to the forecasts of the analytical agency AUTOSTAT, the market for new trucks in Russia in 2020 may collapse by 40-60\% (source: https://www.autostat.ru/infographics/43890/). Automotive Marketing Agency "Russian Automotive Market Research" is considering a baseline scenario with $30 \%$ market reduction.

Using the developed model we analyzed the short-term and medium-term consequences of shocks in products demand in 2020 for KAMAZ in the context of the implementation of state support programs for the company and without them. We made evaluations under the assumption that the characteristics of the mechanism of demand formation $\left(Y^{*} / \eta\right)$, price conditions $(p / y)$ and fixed assets of the company in the considered time period will remain at the base level of 2019. In the given scenario conditions a decrease in demand is interpreted as a proportional decrease in the frequency of sales $\lambda$ in the model. Below are the results of model evaluations of the company's value in relation to current assets (Fig. 3) and the average capacity utilization rate (Fig. 4) of KAMAZ depending on the interest rate on shortterm loans issued to the company. The blue bars reflect the results of the calculations in the base scenario that corresponds to the performance of the company in 2019 (base interest rate 2019 is $r=10 \%$ ). Green and red bars correspond to forecast scenarios of demand collapse in 2020 by 10 and $35 \%$ as against the previous year (2019) respectively. In the absence of state support a 35\% drop in demand (red bars, Fig. 2) is catastrophic and can lead to bankruptcy of the company while the interest rate on loans subsidies at the level of 2019. Given interest rate subsidies the company will remain in 2020 at the limit of profitability (the ratio $\frac{\lambda W(0)}{y \eta}$ is close to 1 ) if the government subsidies will reduce the real interest rate on company loans to no more than $6 \%$ per annum (red bars, $r=4 \%, r=6 \%$, Fig. 3). However even under state subsidized interest payments an unprecedented decrease in capacity utilization rate is expected ( $u$ will be less than $30 \%$ ) that is a potential threat to social stability due to employment reduction (red bars, $r=4 \%, r=6 \%$, Fig. 4).

The scenario with a $10 \%$ drop in demand for the company's products (green bars in Figs. 3, 4) by expert estimates is optimistic and can only be considered in conditions of the government measures of demand support, i.e. with a significant increase in government orders for the company's products. If this 


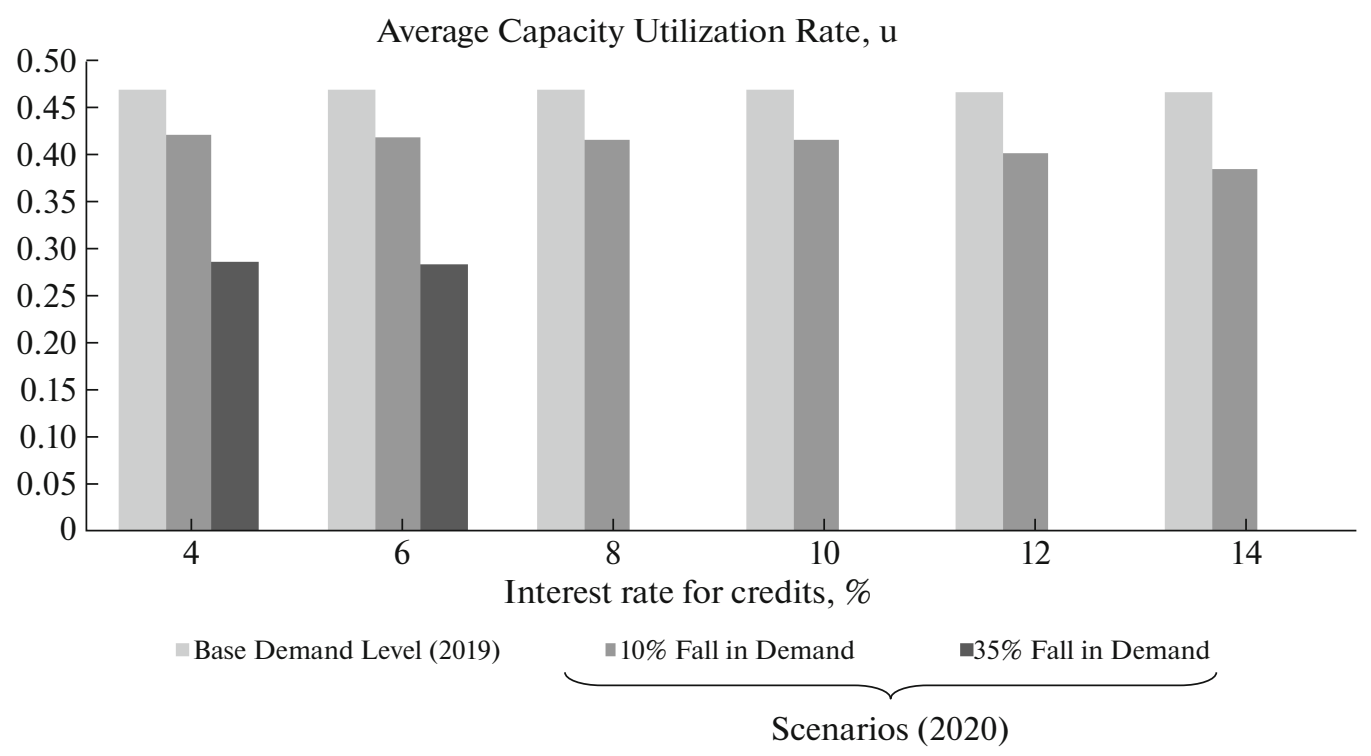

Fig. 4.

scenario is realized while the interest rate remains at the basic level $(r=10 \%)$ then a $30 \%$ decrease in the valuation of company is expected (green bar, $r=10 \%$, Fig. 3 ) as against the 2019 base level (blue bars, $r=10 \%$, Fig. 3). However the company will remain profitable that will allow not to reduce the coefficient of capacity utilization by more than $5 \%$ (green and blue bars, $r=10 \%$, Fig. 4). Thus the adoption of such measures will save the level of employment and will not negatively affect the social stability of the region. Additional measures to subsidize payments on company loans may slightly increase its real value (green bars, Fig. 3). However they will have a negligible effect on the rate of capacity utilization (green bars, Fig. 4).

\section{CONCLUSION}

The calculations show that the application of government measures to increase demand and subsidize interest rates can be effective in the face of a collapse in demand for products of truck manufacturers in 2020. Various options of state support programs for the real sector can be used to support the economy in the context of the COVID-19 pandemic. For example, in a situation where enterprises find themselves on the verge of bankruptcy, one of such measures may be a partial transfer of property rights to the state in exchange for supporting production activities. This approach has been successfully used in the United States during the 2007-2008 crisis. Given the limited state resources, the urgent problem is the selection of the most effective projects for targeted support of enterprises. The developed methodology for the analysis of production performance indicators taking into account the shortage of working capital and unstable demand can be used to analyze and select the most effective measures to support enterprises in the conditions of a collapse in demand caused by a pandemic.

\section{FUNDING}

The research was supported by the Russian Foundation for Basic Research, project no. 20-0700285.

\section{REFERENCES}

1. L. Johansen, Production Functions. An Integration of Micro and Macro Short Run and Long Run Aspects (North-Holland, Amsterdam, 1972).

2. A. A. Shananin, "Investigation of a class of production functions arising in a macrodescription of economic systems," Comput. Math. Math. Phys. 24, 127-134 (1984).

3. W. Hindenbrand, "Short-run production function based on microdata," Econometrica 49, 1095-1125 (1981). 
4. N. K. Obrosova and A. A. Shananin, "A production model in the conditions of instable demand taking into account the influence of trading infrastructure," Dokl. Math. 87, 372-375 (2013).

5. N. K. Obrosova, and A. A. Shananin, "Study of Bellman equation in a production model with unstable demand," Comput. Math. Math. Phys. 54, 1411-1440 (2014).

6. N. K. Obrosova and A. A. Shananin, "Production model in the conditions of unstable demand taking into account the influence of trading infrastructure: Ergodicity and its application," Comput. Math. Math. Phys. 55, 699-723 (2015).

7. D. A. Alimov, "On the existence and uniqueness of a solution of the Bellman equation in a model of operation of a manufacturing company with regard to the debt load," Differ. Equat. 54, 392-400 (2018).

8. D. A. Alimov, N. K. Obrosova, and A. A. Shananin, "Enterprise debts analysis using a mathematical model of production, considering the deficit of current assets," Lobachevskii J. Math. 40 (4), 385-399 (2019).

9. D. A. Alimov, N. K. Obrosova, and A. A. Shananin, "Methodology for assessing the value of an enterprise in the depressed sector of economy based on solving of the Bellman equation," in IFAC Papers-Online Preprints, Proceedings of the 17th IFAC Workshop on Control Applications of Optimization, Yekaterinburg, Russia, October 15-19, 2018 (2018), pp. 788-792. 